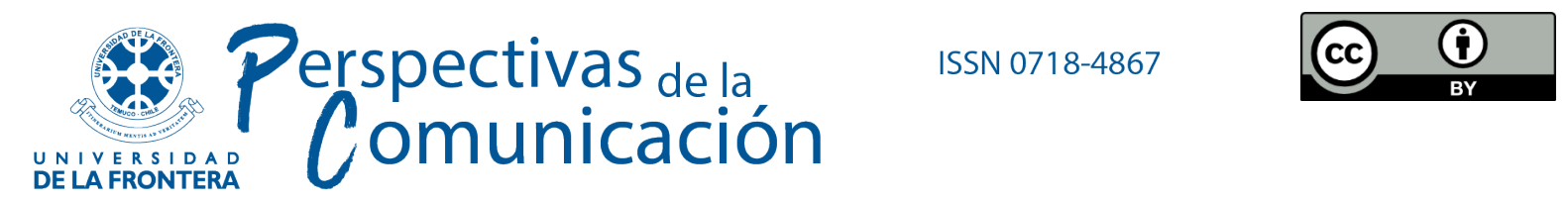

Artículo

\title{
EL INFORMATIVO COMO ORDENADOR EN EL NUEVO ECOSISTEMA DE MEDIOS. NOTICIAS SOBRE DELITOS Y PÚBLICOS EN CUATRO CIUDADES ARGENTINAS.
}

DOI: POR ASIGNAR

\author{
Dra. Brenda Focás \\ CONICET, Universidad Nacional de San Martín, Buenos Aires, Argentina. \\ bfocas@unsam.edu.ar \\ ORCID iD: https://orcid.org/oooo-0002-1749-6757 \\ Dr. Gabriel Kessler \\ CONICET, Universidad Nacional de San Martín, Buenos Aires, Argentina. \\ gabokessler@gmail.com \\ ORCID iD: https://orcid.org/oooo-0003-4204-8961
}

Recibido el 2020-11-30

Revisado el 2021-04-28

Aceptado el 2021-04-28

Publicado el 2021-07-12

\section{Resumen:}

Los informativos nacionales son instituciones tradicionales en las televisiones de todo el mundo. Esta investigación basada en 12 grupos focales sobre los 8 noticieros prime time de las 4 principales ciudades argentinas se pregunta por el rol de estos programas en el ecosistema de medios actual y en particular sobre el consumo de noticias sobre delitos. Nuestro argumento es que los individuos consumen noticias por distintos medios a lo largo del día. Tanto por consumo incidental como por consumo activo, van acopiando temas y atributos sin un orden o jerarquía. El noticiero nocturno tiene el rol de ordenar esas noticias, establecer cierta jerarquía entre los temas, discernir lo verdadero de lo falso. Si bien esto no es privativo de las noticias sobre delito, es particularmente claro en estas noticias, dado la preocupación por el tema como la gran cantidad de noticias sobre tales tópicos. De este 
modo, el noticiero va mutando su rol tradicional y, a pesar de la diversidad de ofertas de medios, refuerza su credibilidad como género informativo y su rol en la construcción de agendas personales y sociales.

Palabras clave: recepción, noticias, consumo de medios, inseguridad. 


\title{
THE TV NEWS AS AN ORGANIZER IN THE NEW MEDIA ECOSYSTEM. CRIME NEWS AND PUBLICS IN FOUR ARGENTINE CITIES.
}

\begin{abstract}
Summary:
The TV news are traditional institutions in televisions around the world. This research based on 12 focus groups on the 8 prime time news in the 4 main Argentine cities examines the role of these programs in the current media ecosystem and on the consumption of crime news. Our argument is that public consume news by different means throughout the day. Both by incidental consumption and active consumption, they are gathering topics and attributes without an order or hierarchy. The prime-time TV news has the role of organizing this news, establishing a certain hierarchy among the topics, discerning what is true from what is false. While this is not exclusive to crime news, it is especially clear in this news, given the concern about the topic and the large amount of crime news. In this way, TV news is modifying its traditional role, and, despite the diversity of media offerings, it is reinforcing its credibility as a reliable institution and its place in the construction of individual and social agendas.
\end{abstract}

Keywords: reception, news, news consumption, crime. 


\section{Introducción}

Los informativos nacionales son instituciones que datan de los comienzos mismos de la televisión en cada país y han contribuido a la conformación de las identidades nacionales (Ponce, 2015; Yorke, 2017). A pesar de los cambios en el ecosistema de medios, en los géneros y el lenguaje televisivo, continúan contando con importante audiencia y credibilidad. Silverstone (1996) señala la relevancia de los noticieros en la definición y el mantenimiento de las rutinas cotidianas y afirma que estos se relacionan, en un punto, no solo por los contenidos de los medios, sino también por "los horarios, géneros y narrativas" (p. 38). Más aún, el género noticiero televisivo, según este autor, permite ver "la articulación dialéctica de angustia y seguridad (...) que opera entre la función de tranquilizar e impactar" (p. 39). Se trata, entonces, de "una institución clave en la mediación de la amenaza, el riesgo y el peligro (...) su significación y su función son tan importantes como lo es su papel proveedor de información" (p. 40). El noticiero, tal como se lo llama en Argentina, se constituye socialmente en el discurso de los acontecimientos, formando hábitos de consumo y modos legitimados de comprensión de la realidad (Pajoni, 2012). Por lo demás, existe una complementariedad entre las formas novedosas de producción de noticias y las más tradicionales.

¿Por qué y para qué las audiencias miran los informativos? En un trabajo pionero en Estados Unidos de los años 70, M. R. Levy (1977) encontraba que las personas buscaban en ellos reaseguro o sensación de control (estoy al tanto de lo que va a suceder), adquirían información sobre temas; intentaban entretenerse y/o reafirmar su insatisfacción o crítica sobre aspectos de la vida social o política. Más recientemente y en Argentina, las y los entrevistados de A. Cantú (2013) adjudicaban al noticiero un rol de mediador entre conflictos en la sociedad, de defensor del pueblo frente a distintos abusos, como una ventana al mundo y lo juzgaban como una empresa con intereses propios.

Los estudios actuales analizan la interacción entre distintos medios y plataformas en la conformación de la agenda mediática, política y social, tanto la de primer nivel (qué temas pensar) como de segundo nivel (con qué atributos se presenta cada tema) (Andrews \& Caren, 2010; Feezell, 2018, Harder, Sevenans \& Van-Aelst, 2017). Sin embargo, no contamos aún con muchos estudios recientes sobre el rol de los informativos en este contexto novedoso y menos aún en el caso de América Latina. Nos preguntamos entonces para sus públicos cuáles funciones tradicionales de los informativos se mantienen y cuáles otras han cambiado. Nuestro estudio de recepción de noticias en general y de delitos en particular nos lleva a 
sostener el siguiente argumento. Los individuos consumen noticias por distintos medios a lo largo del día, una gran parte de ellas sobre delitos, el eje de nuestra investigación. Tanto por consumo incidental como por consumo activo, van acopiando temas y atributos sin un orden o jerarquía. El noticiero nocturno tiene el rol de ordenar esas noticias, establecer cierta jerarquía entre los temas, discernir lo verdadero de lo falso. Si bien esto no es privativo de las noticias sobre delito, es particularmente claro allí, dada la preocupación por el tema como la gran cantidad de noticias sobre tales tópicos. Esta necesidad de ordenamiento guarda relación directa con el mayor consumo de noticias sobre delitos, en particular en sectores populares de la periferia de Buenos Aires, que aunaban alta preocupación por el tema con mucho tiempo de viajes diarios a sus trabajos durante los cuales estaban expuestos a medios. De manera casi contra intuitiva, cuando más información de todo tipo circula, más parece demandarse el rol del noticiero poniendo orden en ese "sinfín" de noticias, tal como lo llamaban.

El presente artículo se basa en los resultados de doce grupos focales de un proyecto de análisis integral de producción y consumo de noticias de delitos en los noticieros prime time de la Argentina. En primer lugar, luego del apartado metodológico y teórico, nos concentramos en los cambios en las formas de recepción, a continuación, en la relación entre el consumo incidental y el activo, para finalmente indagar en el rol del noticiero en este nuevo ecosistema informativo.

\section{Estrategia metodológica y corpus de análisis}

Este artículo forma parte de dos proyectos de investigación que analizaron el circuito informativo de las noticias de delito e inseguridad, de manera integral ${ }^{1}$. En la instancia de la recepción, la estrategia para indagar esta hipótesis se basó en la técnica de grupos focales en 4 ciudades argentinas: Ciudad de Buenos Aires y área metropolitana del conurbano, Rosario, Córdoba y Mendoza ${ }^{2}$. Se trata de una metodología que suele ser útil para elucidar contenidos

1El artículo se desprende de los proyectos: PICT y PIO- CONICET- Defensoría del Público: "De la propiedad a la recepción. Estudio integral del circuito productivo de las noticias sobre delito e inseguridad en los noticieros televisivos de mayor audiencia de la Argentina”.

2 Buenos Aires es la capital del país y cuenta en 2020 con más de 3 millones de habitantes y tiene estatus de ciudad autónoma (Ciudad Autónoma de Buenos Aires o CABA por sus siglas). El Gran Buenos Aires (GBA) reúne 24 distritos del conurbano de la ciudad que suman casi 10 millones de habitantes pertenecientes a la Provincia de Buenos Aires. CABA y GBA conforman el 
propios de un grupo determinado y se presta para el análisis de recepción mejor que las encuestas o entrevistas individuales. Al proponer una perspectiva integral en el estudio de las fases del proceso productivo de las noticias para contribuir a su comprensión, el proyecto elaboró un corpus de análisis con los noticieros transmitidos durante el horario central por los canales 11 y 13 emitidos desde la Ciudad Autónoma de Buenos Aires, 10 y 12 de Córdoba, 3 y 5 de Rosario y 9 y 7 de Mendoza33, en el período de dos meses (mayo y junio de 2016).

En este artículo abordaremos la instancia de la recepción para la cual trabajamos con doce grupos focales conformados por diez personas que fueron distribuidas según edad, clase social y lugar de residencia. Dentro de los noticieros señalados, seleccionamos noticias de delito, violencia e inseguridad para exponer a los participantes entendiendo tanto los delitos de distinto tipo como aquello que es integrado dentro de la noción de inseguridad, aunque no implique necesariamente un conflicto con la ley. La primera, emitida por el noticiero de Telefé Noticias, narraba un robo en un supermercado con propietarios de origen chino donde un policía, que custodiaba el lugar, mató al presunto ladrón. Lo particular de esa noticia es que aparecía el cuerpo del asesinado4. La segunda, emitida por Telenoche, versaba sobre un caso de corrupción (Lázaro Báez)5. La última, emitida por canal 10 de Córdoba, abordaba un caso de femicidio (Carina Drigani) ${ }^{6}$. Se procuró que hubiera diversidad de temas vinculados con el delito y la inseguridad y de canales de televisión, para analizar si alguna de esas variables incidía en los modos de interpretación de las noticias. Para trabajar

área metropolitana de Buenos Aires (AMBA) que no tiene estatus jurisdiccional propio. La ciudad de Córdoba (1.450.0oo habitantes) es la capital de la provincia homónima en el centro-norte del país, Rosario (1.200.00o) en el centro es la principal ciudad (pero no la capital) de Santa Fe y Mendoza en el oeste es la capital de dicha provincia y junto al Gran Mendoza cuenta con una población mayor al millón de personas. Se trata de los 4 principales centros urbanos del país.

$3 \mathrm{Si}$ bien el rating es cambiante, los noticieros suelen encontrarse dentro de los programas con más audiencias y en particular en las provincias. A modo de ejemplo, en Córdoba los dos informativos estudiados estaban primero y tercero en el rating (ver: https://vos.lavoz.com.ar/tv/los-50-programas-locales-mas-vistos-en-cordoba) y el noticiero de Telefe en el momento de escribir esta introducción era el tercer programa más visto. https://www.primiciasya.com/rating

4 Robo y muerte en "supermercado chino" Canal: Telefé. Fecha de emisión: 02/o8/2016. Duración: 4 minutos 59 segundos. Primer bloque. Más información sobre la noticia: ttps://telefenoticias.com.ar/actualidad/impactante-video-del-momento-enque-un-policia-mato-a-un-ladron-en-un-supermercado-chino/

5 Lázaro Báez. Canal: Canal 13. Fecha de emisión: 1/o8/2016. Duración: 6 minutos 1 segundo. Primer bloque. Más información sobre la noticia: https://www.infobae.com/politica/2016/o7/03/la-entrevista-completa-a-lazaro-baez-desde-la-carcel/

6 Femicidio Carina Drigani. Canal 10 de Córdoba. Fecha de emisión: 2/o8/2016. Duración: 11 minutos 11 segundos. Primer bloque. Más información sobre esta noticia: https://www.lanacion.com.ar/seguridad/horror-en-cordoba-hallan-muerta-a-lafisioterapeuta-que-estaba-desaparecida-nid1895965 
en los grupos diseñamos una guía de pautas para poner a prueba distintas hipótesis y hallazgos internacionales sobre recepción de noticias. También realizamos una encuesta cerrada sobre consumo de información que fue entregado a los participantes antes de comenzar con la dinámica de los grupos. El análisis de los grupos siguió un abordaje comparativo sobre similitudes y diferencias en los tópicos indagados a partir las variables preestablecidas (sexo, grupo etario, nivel socioeconómico y ciudad)7, así como otras que fueron surgiendo en el tratamiento de datos en particular vinculados al tipo de consumo de medios y de noticias.

\section{Resultados}

\subsection{Cambios en la recepción de noticias}

La pregunta por la recepción y el consumo de noticias ha ocupado un lugar relevante en el campo de los estudios de Comunicación en América Latina. En general se cuestiona la incidencia de los medios en la configuración de problemas sociales que acaparan y direccionan la opinión pública sobre ciertos temas. Se sostiene que exageran en la enunciación de las noticias, que tienen intereses o intenciones en generar miedo, que son sensacionalistas. En la literatura hay acuerdo en que los medios aportan una mirada sobre ciertos temas, colaboran en la legitimación de ciertas creencias, y proveen temas y tópicos para la discusión de agendas. Es decir, se trata de actores que, mediante procesos de selección, omisión y jerarquización les otorgan visibilidad a ciertos temas y les restan visibilidad a otros (McCombs, 2007). Sin embargo, hay menos consenso acerca de la

\footnotetext{
7 Específicamente los 12 grupos fueron segmentados de la siguiente manera. En Ciudad de Buenos y área metropolitana se realizaron 6 grupos de 10 personas cada uno. 1. 18-30 años. NSE C1-C2. Residentes en CABA, todos los barrios, menos Sur. 2. 40-65 años. NSE C1 y C2. Residentes en CABA, todos los barrios, menos Sur. 3. 20-50 años. NSE D1, D2. Residentes en barrios Sur de la CABA (Lugano, Soldati, Barracas, Boca, Constitución, Floresta si bien no es Sur, Balvanera). 4. 30-50 años. NSE C1 C2. Residentes en GBA primer cordón, Norte, Sur y Oeste. 5. 18-30 años. NSE D1- D2. Residentes en GBA segundo y tercer cordón. 6. 40-56 años. NSE D1 D2. Residentes en GBA segundo y tercer cordón. En las ciudades de Córdoba, Rosario y Mendoza trabajamos con 2 grupos en cada ciudad Grupo 1: 25 a 55 años. NSE bajo, y el otro grupo de 25 a 55 años NSE medioalto. Los participantes fueron en partes iguales varones y mujeres, fueron reclutados por personal especializado mediante método bola de nieve, asegurando que no se conocieran entre ellos y que hubiera en cada grupo diversidad etaria, de inserción laboral y que provinieran de distintas zonas o barrios de cada ciudad.
} 
recepción, pregunta que se ha complejizado a partir de los cambios en el escenario mediático intensificados por la digitalización.

Una primera cuestión que se debe considerar para analizar la práctica del consumo de medios es el cambio en el ecosistema informacional. El actual panorama se caracteriza por la pérdida de una agenda común entre medios y público debido a la multiplicación de la oferta, a audiencias multitasking -esto es, que están expuestas a una variedad de medios al mismo tiempo- y que a la vez son productoras de contenido periodístico (Kessler \& Focás, 2014). A esto se suma el crecimiento de los medios virtuales, con noticias que cambian minuto a minuto en los portales, la proliferación de información en las redes sociales, y en WhatsApp, lo que lleva a un consumo de la información más ecléctico y veloz. La convergencia basada en la digitalización modificó las lógicas de producción, distribución y consumos en los medios, e impactó en la necesidad de reconfigurar oferta de contenidos informativos y de entretenimientos para adecuarse a las nuevas demandas (Becerra \& Marino, 2021) ${ }^{8}$.

Estamos, sin dudas, ante un nuevo consumidor multimediático, receptor de diferentes medios, por un lado, y a la vez productor de información que circula por los dispositivos tecnológicos y que disputa con el periodismo profesional su tradicional monopolio de la veracidad sobre los hechos (Boczkowski \& Mitchelstein, 2013; Amado \& Rincón, 2015; Lee \& Tandoc, 2017)._A diferencia de públicos fragmentados, cada uno dentro de su "cámara de eco" (Berry \& Sobieraj, 2014), encontramos en este caso una tendencia a la duplicación y superposición de audiencias, tal como sugieren algunos autores que señalan una "cultura masivamente superpuesta" a pesar de la creciente oferta mediática (Webster, 2005, 2014).

En relación con sus hábitos informativos, las y los participantes de los grupos focales concordaban que leen noticias por medios digitales y redes sociales, mientras que la televisión sigue ocupando un lugar importante en la práctica de informarse.

8 La concentración de medios es muy alta en el país, lo que incide en las construcciones noticiosas y sus audiencias. Por caso, el Proyecto Media Ownership Monitor (MOM, 2019) arroja un 59,24\% de concentración por parte de los 8 principales grupos y un cuarto del total de las audiencias en todos los medios usa/consume productos del Grupo Clarín. Para más información ver: Becerra \& Marino (2021). 
Yo más que nada por Internet, el celular mucho... más que nada viendo por ahí algunas páginas de Facebook que por ahí orientan o Google Si no bueno, el noticiero. Diarios no mucho.

(M, NSE medio, Rosario 25-30)

Cuando pasa algo lo primero que es inmediato es Internet, uno agarra el teléfono y enseguida se entera lo que pasó antes que en la tele o en el noticiero.

(H, NSE medio, Mendoza 25-30)

Todo lo que veo en Facebook es por gente conocida que comparte.

(H, NSE bajo, GBA 18-30)

En Google me aparece si quiero recibir noticias de donde estoy. Entonces por ejemplo ahora vine para Capital y me llegan noticias de ahí entonces las miro así rápido a ver si pasó algo...

(M, NSE bajo, GBA 1er cordón, 30-50)

Los testimonios muestran que las redes sociales y el uso del smartphone modificaron sustancialmente los modos de consumo de información de las audiencias. En general el primer contacto con las noticias comienza desde los dispositivos donde acceden a información mediante las redes sociales y luego, entran a los portales. En una encuesta que realizamos con los participantes de los grupos focales ${ }^{9}$ se desprende que la televisión sigue siendo el principal medio para informarse, aunque la distancia en relación con el uso de medios digitales y redes para este fin es mínima (ver Gráfico 1).

9 Tal como se describe en el apartado metodológico, antes de comenzar la realización de los grupos focales, las y los participantes respondieron una encuesta cerrada sobre consumo de medios. En total se realizaron 120 encuestas en las cuatro ciudades del país. 
Gráfico 1. ¿Por qué medios se informa?

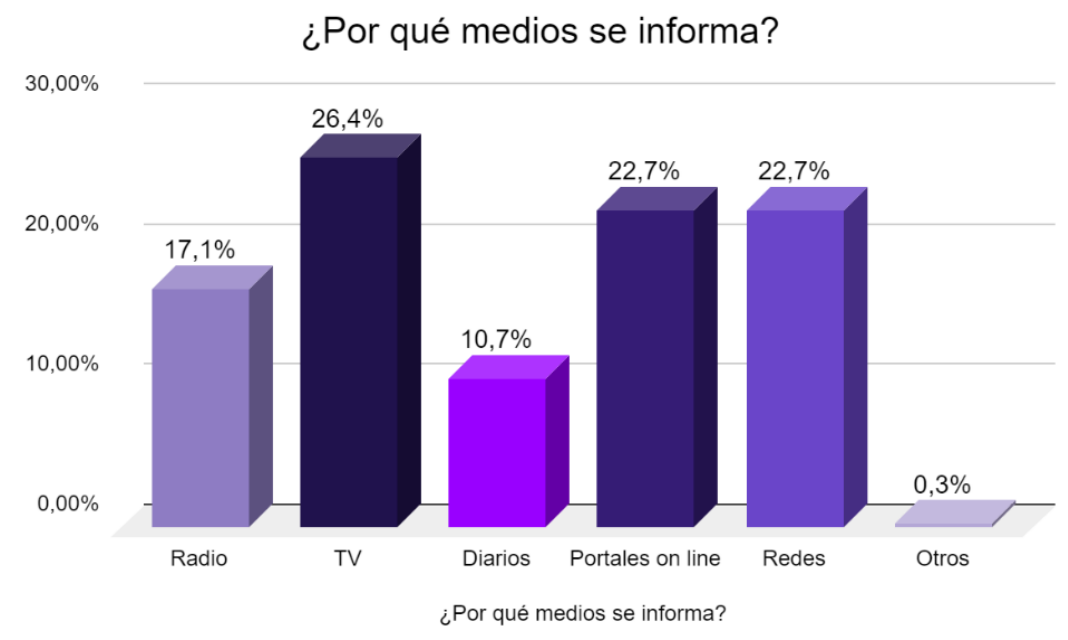

Fuente: Elaboración propia en base a encuestas cerradas con participantes de los grupos focales.

Los participantes de los grupos, en general, consumen información desde dispositivos variados y en distintos momentos del día. La televisión por aire y por cable e Internet fueron las plataformas más elegidas, mientras que la radio y la lectura de diarios impresos ocupaba un lugar menor en sus prácticas ${ }^{10}$.

Los más jóvenes realizan la lectura de noticias a través de las redes sociales en Internet, en especial Facebook. También usan Instagram y Snapchat y, en menor medida, Twitter. Las noticias se entremezclan con los posteos propios de las redes y, de esa forma, logran captar el interés de los jóvenes. Muchas veces a partir de este primer acercamiento, si alguna noticia les interesa, visitan los portales de los diarios online para acceder a más información, tema que abordaremos más adelante. Falta sin duda en el contexto local un análisis más detallado de la forma de consumo por móviles, como la diferencia que encuentran Nelson \& Lei (2018)

10 De acuerdo con el informe del Instituto Reuters de la Universidad de Oxford (2020) Internet y la televisión siguen siendo las fuentes de noticias más populares en Argentina, mientras que el consumo semanal de la prensa escrita cayó del $45 \%$ al 23\% en los últimos cuatro años. La mayoría de los encuestados dijeron que usan el teléfono con acceso a internet para acceder a las noticias. 
entre consumo intenso de noticias y más tenue, según si se siguen aplicaciones de medios de noticias en el primer caso o se llegan, como en el segundo tipo, a los medios digitales por medio de buscadores.

Una segunda cuestión que considerar es el tiempo que se le dedica al consumo de información. Los estudios clásicos de recepción hacían hincapié en el consumo hogareño, específicamente en las prácticas y rituales que se generaban alrededor del uso de la televisión (Morley, 1996; Silverstone, 2004). Hoy, no existe un tiempo determinado dedicado a mirar noticieros, o leer el diario, sino que las noticias son recibidas todo el tiempo, entremezcladas con otras interacciones como el uso del WhatsApp, revisar los mails, o googlear. Estudios indagaron en el cruce entre el uso del tiempo y la recepción en distintos soportes (Raeymaeckers, 2002; Julsrud \& Denstadli, 2017), y en relación con la cantidad de tiempo frente a dispositivos y sus posibles efectos nocivos (Alghadir, Gabr, \& Iqbal, 2016) y los cambios que implicó en el consumo de medios el uso de dispositivos móviles (Thurman, 2017; Lyons \& Urry, 2005).

En nuestro trabajo encontramos que la práctica varía en relación con las ocupaciones diarias, es decir, no hay un tiempo fijo para informarse. Puede ser mirar los portales en algún momento de la jornada laboral, al mediodía con la televisión, e incluso escuchar la radio en los trayectos de un lado a otro. Lo cierto es que las y los participantes de los grupos consumen información durante gran parte del día desde distintos dispositivos, incluso sin tener la necesidad o el interés por informarse. Una particularidad tiene lugar, por ejemplo, en los grupos de participantes que vivían en el Conurbano de la ciudad de Buenos Aires y viajaban entre dos y cuatro horas diarias para llegar a la Capital para trabajar o estudiar, tal como muestran estos testimonios:

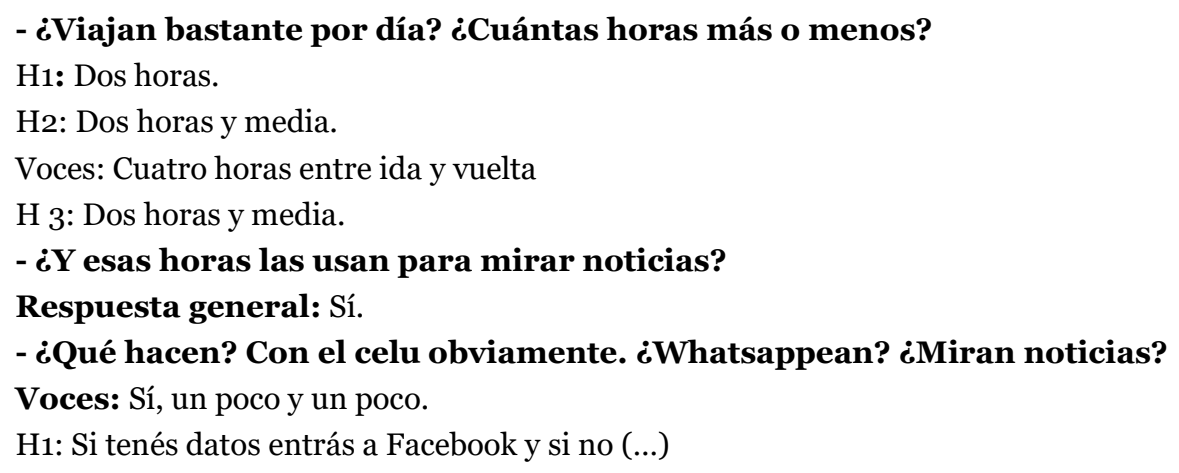


Investigaciones empíricas mostraron que el acceso a la comunicación móvil durante los viajes en transporte público influye en cómo los pasajeros usan ese tiempo de viaje. Actividades que estaban relacionadas con un espacio definido como el trabajo, la educación y el ocio, ahora son realizadas en el tiempo de viaje (Lenz \& Nobis, 2007; Bayarma, Ettema \& Dijst, 2010). El transporte público se convirtió en el "lugar" donde se realizan diferentes actividades, y es una oportunidad para realizar múltiples tareas con diferentes grados de complejidad (Kenyon \& Lyons, 2007; Guo, Derian \& Zhao, 2015). La comunicación digital es una aliada del consumo en los viajes ya que en el transporte público se "liberan las manos del volante", usándolas en los dispositivos (Urry, 2007). El lugar de acceso a los contenidos de las noticias se ha desplazado de la casa y el trabajo a todas partes y a ninguna en particular al mismo tiempo. El consumo de noticias en pantallas se "intercala a lo largo del día sin la previsibilidad que caracterizaba el acceso a las noticias en los diarios impresos, la radio, la televisión y las computadoras" (Boczkowski, Mitchelstein \& Matassi, 2017, p. 1790), por lo que el tiempo de viaje no es un tiempo perdido, tal como muestran los testimonios:

\footnotetext{
- ¿El viaje es un momento donde uno se informa bastante de lo que está pasando? Voces afirmativas

H1: sí, yo veo portales

H2: FM. Escucho a María O’ Donnell

M: yo, Twitter, WhatsApp

- ¿Se informan por Whatsapp, por los grupos?

[dicen que sí varios]

H1: sí, por ejemplo, inundaciones, como pasó ahora que el viento subió la marea, y yo que estoy en el Tigre...

(Grupo focal, NSE bajo, GBA, segundo y tercer cordón, 40-56)
}

Las y los participantes dan cuenta de las formas en que ha variado la recepción de noticias y cómo el tiempo de viaje de transporte público es utilizado, entre otras cosas, para informarse. Del mismo modo, Ferrante (2019) muestra que las y los pasajeros del metro de Buenos Aires, usan sus celulares para acortar el tiempo y hacer más ameno el viaje. Para los pasajeros es un modo de ahorrar tiempo (son contenidos que verían en otro momento, en otro espacio), refugiarse del entorno y mantenerse informados. En síntesis, dentro de los cambios en los modos de consumo, observamos que la digitalización y la extensión del uso de dispositivos móviles permitió el acceso a mayores fuentes de información. El tiempo de viaje, es uno de los momentos en que más se utilizan los celulares tanto para ocio como para 
informarse, y en particular observamos que quienes tienen mucho tiempo de viaje como quienes viajan desde el conurbano a la ciudad para trabajar, aprovechan estos dispositivos para múltiples tareas, entre ellas informarse. Incluso las redes sociales funcionan hoy como un modo de acceso a las noticias, muchas veces de forma incidental, como veremos en el próximo apartado.

\subsection{Del consumo incidental al activo}

La práctica de utilizar el smartphone constantemente lleva a que muchas veces las personas se informen, sin tener esta actividad como primera intención. Es decir, los sujetos se conectan a las redes sociales para mirar fotos y/o posteos de amigos y en ese recorrido se encuentran con noticias. Puede ser que alguien posteó información sobre un caso, por alguna cadena por WhatsApp, incluso por algoritmos por los que se sugiere la lectura de algunas noticias en medio de todo. Esa forma de decodificar la información se denomina "consumo incidental", esto es el consumo fortuito, o azaroso de noticias. Según Mitchelstein y Boczkowski (2017), en los últimos años este tipo de acceso al contenido de actualidad dejó de ser periférico para convertirse en central y primario, sobre todo en los usuarios más jóvenes y conectados. El estudio también muestra, en consonancia con nuestras observaciones, que "el acceso incidental a la información en redes sociales más que reemplazar, complementa los hábitos informativos preexistentes” (p. 140). En los grupos las y los participantes dieron cuenta de que consumen información de forma incidental:

\section{- ¿Siguen alguna página de noticieros?}

(Algunos responden "sí")

H1: Yo no sigo ninguna. Todo lo que veo en Facebook es por gente conocida que comparte.

M1: En Google me aparece si quiero recibir noticias de dónde estoy. Entonces, por ejemplo, ahora vine para Capital y me llegan como noticias de ahí entonces las miro así rápido a ver si pasó algo...no sé.

- ¿Por dónde las ven?

H2: sobre todo en Facebook de gente que comparte cosas, cada tanto entro así a alguna página o a algún portal de noticieros de, de noticieros no, de diarios, Tiempo Argentino, Página 12.

(Grupo focal, NSE medio GBA primer cordón, 30-50)

Básicamente me voy enterando en las charlas. Escucho radio, me voy enterando lo que ocurre cotidianamente en la radio, en Facebook también, pero depende de lo que compartan mis amigos. Y me junto con la gente del barrio y dicen che, ¿viste secuestraron a dos chicas?

(H, NSE medio, CABA,18-30)

Perspectivas de la Comunicación - Vol. 14 - No 1 - 2021 - pp. 139-168

Universidad de la Frontera - Chile 
Los testimonios muestran que "las noticias se les aparecen", sin una necesidad o interés previo por informarse. El consumo incidental es cada vez más frecuente en las redes sociales y los algoritmos cumplen un rol importante llamando la atención sobre noticias que de otra forma hubiesen pasado desapercibidas por las audiencias. Estos no solo actúan como gatekeepers, sino que también, junto con los nuevos líderes de opinión, ofrecen encuadres que promueven ciertas interpretaciones de las audiencias (Bergström \& Jervelycke, 2018). Nuestros entrevistados dan cuenta de las formas en que cambiaron los procesos de recepción de noticias. Se accede desde distintos dispositivos, y entre juegos, ocio y redes sociales aparece la información. Esta práctica la observamos en todos los grupos, tanto en los de sectores medios como bajos, así como tampoco encontramos diferencias en relación con la edad. Ahora bien, una vez que la información llega, el interés varía en relación con el tema, y el abordaje y se abren distintos caminos luego de este primer contacto incidental. Wieland \& Kleinen-Von-Königslöw (2020) proponen tres caminos posibles, luego del primer contacto incidental. Un camino activo es aquel en el que un encuentro incidental con las noticias lleva a un compromiso activo con ellas, por ejemplo, leer un artículo completo; sin embargo, al menos son posibles otros dos caminos: seguir un camino automático (desplazarse por el suministro de noticias o hacer scrolling) o un camino incidental breve ("picotear las noticias", leer solo los títulos o hasta el primer párrafo, por ejemplo).

Tomando esta propuesta, notamos que una particularidad de las noticias es que con frecuencia ese consumo incidental (desinteresado) suele transformarse en un "consumo activo" cuando se trata de tópicos vinculados con lo delictivo. Si bien las y los participantes comenzaban de forma aleatoria mirando los feeds o los portales, los temas de delito son los que suelen captar más su atención, como muestran estos testimonios:

\footnotetext{
- ¿Por qué las miran?

M: sí, para prevenir.

V: Para saber dónde fue también, el lugar.

M: claro el lugar también.

(Grupo focal, NSE bajo, Rosario 25-55)
}

-Cuenten quiénes miran, qué miran. Los que miran el celular, ¿qué miran?

M: No sé si es porque yo estoy como medio fanática, dicen mataron a una mujer y me gusta seguir todo el caso, viste, y busco y busco,

¿En el celular? 
M: Busco con el celular, por ejemplo, y es como acá dicen mataron a esta mujer ahorcada, lo dan ponele un noticiero. En la otra dicen la ahorcaron y la acuchillaron; no dicen todo en uno solo. En otro dice ya no la ahorcaron, fue estrangulada o fue golpeada.

\section{¿Y dónde buscan? ¿En páginas distintas?}

M: No, yo por ejemplo, yo estoy en el Face y está el título y vos entrás a ése y mientras vos vas viendo la noticia van apareciendo otras noticias. Y después volvés a entrar, digo voy a ver qué información, hay más información.
Ah, te sugiere eso. Uno mira eso y ya la máquina...
M: Y cuando vos vas pasando van saliendo, te van saliendo.
M: Te van sugiriendo con los títulos.
$\mathrm{H}$ : O tiene comentarios la misma noticia...
(Grupo focal, NSE bajo, Córdoba, 25-55)

Por último, una cuestión que se desprende del consumo activo es que, una vez que una noticia genera interés, hay una práctica extendida en los usuarios que consiste en buscar más información en otros medios. Esta práctica, que se acentúa cuando una noticia delictiva les interesa, les permite "armarse su propia noticia". Observamos un quiebre donde se pierde la fidelidad a un medio determinado, y el proceso de recepción se realiza buceando sobre la misma información, pero contada desde diferentes miradas, como cuentan estos participantes:

M: Sí, yo lo mismo, miro la televisión, los noticieros, me mato con los noticieros. No sé por qué pero me encantan. Y el tema de Internet también y hago comparaciones, lo que dice un lado, lo que dice el otro, para ver si...

\section{Por ejemplo ¿qué comparás?}

M: No, lo que sale en alguna noticia y lo busco en otra página para ver si dice lo mismo...

M2: Lo que pasa es que cada uno lo ve con su visión. Hay algunas cosas que sí, que coinciden y unas cosas que uno dice una cosa y el otro dice cosas completamente distintas.

(Grupo focal, NSE bajo, 30-50 años, GBA)

Los testimonios muestran que en general las y los participantes valoran el pluralismo, consideran la realidad compleja y/o difícil de comprender y en consecuencia intentan formarse un juicio propio a partir de distintas fuentes cuando un tema les interesa (Focas, 2020). Es decir, el proceso de recepción y de interpretación de la información se realiza buceando sobre la misma información, pero contada desde diferentes miradas.

En síntesis, la literatura encuentra evidencia acerca del consumo incidental como una modalidad común de acceso a las noticias. Lo novedoso en esta investigación es que las 
noticias sobre delitos suelen captar la atención y transformar ese consumo incidental en activo. Hay particularidades, como que las mujeres se interesen más por los femicidios, o temas de violencia de género. Pero también existen otros mecanismos que activan el interés como el rol pedagógico de las noticias de inseguridad que se evidencia en el hecho de que los públicos utilizan en su vida cotidiana información vinculada con la inseguridad, tanto como modo de prevención o como ayuda para configurar una cartografía del delito (Focas, 2020). Los algoritmos, también cumplen una función importante en guiar estas lecturas y por último hay en el consumo de estas noticias cierto "morbo" como veremos más adelante.

\subsection{El noticiero como ordenador}

Más de la mitad de las y los participantes de los grupos afirmaban mirar el noticiero de la noche, con mayor frecuencia en los grupos de edad mayores, quienes duplicaban en su consumo al grupo de 18 a 26 (del 36\% al $68 \%$ en los de 51 a 65). ¿Por qué luego de pasar todo un día envuelto en un sinfín de noticias de todo tipo y de delitos en particular se decide mirar un noticiero? Justamente para ordenar la información recibida a lo largo del día; seleccionar y jerarquizar las noticias por su mayor o menor importancia, discernir entre datos verdaderos y falsos, descartando algunas. La gran mayoría de nuestros entrevistados coinciden en que en los noticieros rige un criterio de selección de las noticias ligadas a la relevancia: su misión es presentar en un tiempo limitado los principales hechos que sucedieron en la jornada y así ofrecer al final del día la posibilidad personal de poner organizar ese flujo desordenado y sin criterios de relevancia que se fue conformando por múltiples canales a lo largo del día, como se desprende de los testimonios:

Uno sigue el desarrollo de una información porque uno siempre escucha me enteré por ahí que, no sé bien, ...entonces uno cuando lo ve por los canales oficiales de distribución de información más o menos va creyendo, descreyendo, de las informaciones que uno tiene.

(M, NSE bajo, Rosario 25-55)

H: La diferencia, vos preguntabas la diferencia entre las redes...no, que uno es más rápido y bueno, como decís vos, por ahí lo ves y no lo creés, te tomás el tiempo para ver si es cierto y por ahí como que el noticiero confirma un poco.

M: Claro, porque hay una persona que te lo está narrando, te está mostrando hechos entonces como que uno le cree más

H2: Claro, aparte el periodista muchas veces refresca, como decía él, dijeron tal cosa y eso es bueno. Las redes no te hacen eso eh.

(Rosario NSE medio, 25-55) 
Cada fragmento de entrevista da cuenta de un aspecto del lugar actual de los noticieros: seleccionar los eventos más significativos y a diferencia de los programas políticos, limitarse a narrar lo que sucedió, ayudando así a "creer y descreer" en la medida que se reafirme o refute información, mostrando los hechos y "refrescando" datos del tema en cuestión. En rigor, nadie le exige al informativo que recupere su rol tradicional de proporcionar primicias. $\mathrm{Al}$ fin de cuentas, las audiencias abordan el noticiero con mayor caudal de información que las que van a presentarse, están al tanto de casi todas ellas, al menos en relación con el delito y, el noticiero les sirve para ordenarlas y proponer un encuadre plausible para cada una de ellas. En los grupos observamos que cuanto mayor es la exposición informativa a lo largo del día, más se demanda al noticiero que funcione como ordenador; esto era particularmente elocuente entre los grupos de nivel socioeconómico bajo de la periferia de Buenos Aires caracterizados por un alto consumo de noticias de delitos en el día. Esto se debe a que se trata de un tema que les preocupa y porque, como vimos, la mayoría pasan varias horas por día en transporte público para ir y volver a los trabajos en el centro de la ciudad.

¿Qué atributos permitirían a los informativos cumplir su misión? En primer lugar, se los considera menos politizados, ideológicos y/o polarizados que los periódicos o canales de noticias de cable. El noticiero es una institución tradicional que se mantiene en todos los grupos con cierta distancia de la crítica demoledora a las mentiras de la política, la banalización de los géneros televisivos o de una polarización, como sí lo harían los diarios y las redes sociales. Así lo expresan testimonios de diferentes grupos e individuos con distintas tendencias políticas:

Yo enojarme con el noticiero, no. Pero a veces cuando leo diarios, porque, por ejemplo, que la corriente política está muy marcada, sí me enoja porque capaz, no sé, tiro dos diarios cualesquiera, leo Clarín y leo Página/12 y dicen lo mismo pero al revés y es como que no te creo ni a vos ni a vos; no sé qué hacer ahora, pero en sí el noticiero, como que en general no es muy político, es más sobre cosas que pasan en la calle o cosas así, como mucho me genera tristeza

(M, NSE medio, Caba 18-30)

El noticiero no "inventa" y esto es particularmente claro en relación con los crímenes: la materialidad de un cuerpo muerto en el caso de los homicidios se impone como una realidad innegable.

Igual lo que es homicidio no creo que, o sea, te pueden inventar otras cosas. O sea, yo no necesito ver un video para creer, no van a salir a inventar porque además hay un tema legal, hay nombres, hay

Perspectivas de la Comunicación - Vol. 14 - No 1 - 2021 - pp. 139-168

Universidad de la Frontera - Chile 
imágenes, el tema legal que vos estás filmando una dirección o nombrás una persona y si es mentira obviamente que después legalmente el canal o el periodista se va a tener que hacer cargo, no es tan fácil inventar algo.

(M, NSE medio, Gran Buenos Aires, 30-50)

Salvo que más allá de la prueba, hay una verdad. Yo fui y maté a esta persona, fui y la maté no hay otra verdad.

(H, NSE bajo, Mendoza 25-55)

El cambio en el ecosistema de medios y en las audiencias, modifica la función habitual de los noticieros y esto plantea interrogantes, entre otros, a los estudios sobre agenda. La audiencia mira el noticiero de la noche con un esbozo de agenda propia conformada a lo largo del día. El informativo gravita tanto en la llamada agenda de primer nivel, en las temáticas sobre las cuales pensar, como en la de segundo nivel, los atributos de los temas (McCombs, 2007). Pero como hemos sostenido, brindando herramientas para reordenar las agendas de primer y segundo nivel en conformación. De algún modo, completa un trabajo diario de conformación de agenda. ¿̇e trata entonces de una manifestación del modelo de agenda en red? Nos referimos a la hipótesis de varios autores quienes afirman que los medios de comunicación tienen la capacidad de construir las conexiones entre las agendas, y de esta forma construir la centralidad de determinados elementos de la agenda en la mente del público y, la prominencia se puede definir como la centralidad de un objeto y/o atributo en la agenda pública (McCombs \& Barrios, 2012; Gou, 2014). En parte sucede, pero es una red en la cual se le adjudica al noticiero una mayor jerarquía que otras fuentes.

Ahora bien, también se miran noticias de delito por otras razones, algunas ya conocidas como la espectacularidad o, como se decía en los grupos, el "morbo" que generan. Lo definían como la atracción por lo prohibido, con un abierto paralelismo con temas sexuales: "cuando más prohibido más te atrae". El tema apareció porque en una de las noticias las cámaras de seguridad mostraban cuando un policía mataba a un ladrón en el supermercado. La discusión sobre si estaba bien o no mostrar a alguien muriendo, llevó a la mayoría de los participantes admitir, con cierto pudor, la atracción de ese tipo de imágenes, asociadas a lo que llamaban "morbo". Cabe señalar que no es habitual ni está socialmente aceptado desde el fin de la última dictadura militar (1976-1983) que se muestre un cuerpo muerto ni en la prensa gráfica ni en la televisión argentina (Gayol \& Kessler, 2018). Así las cosas, el cuerpo muerto de la víctima, podía ser considerado un exceso, pero era una prueba de su veracidad. Por su parte, la noción del sensacionalismo fue utilizada sólo en algunos casos para, por 
ejemplo, criticar el formato (música, voz en off) con que se presentaban las confesiones en la cárcel de un acusado de ser testaferro de los expresidentes Néstor Kirchner y Cristina Fernández de Kirchner. En este caso, tal como lo sostienen Grabe, Zhou y Barnett (2001), el sensacionalismo no estaba ligado al contenido de las noticias, sino al formato y en tal sentido, si afectaba la credibilidad de la noticia.

La credibilidad de los noticieros está en parte determinada por los modos de presentación de las noticias. El análisis de la recepción de noticias sugiere que hay una gramática de producción y otra de recepción en cuanto que hay reglas que no son necesariamente fáciles de expresar, pero sí se advierten cuando se quiebran. En concreto, una noticia debe tener una duración ni tan corta como para ser considerada superficial ni tan larga como para aburrir, una estética atractiva pero acorde a la gravedad del tema en los casos del delito, el relato tiene que ser ameno, pero no puede parecer una ficción. Esto se veía claramente en la noticia visualizada sobre el femicidio de un canal de Córdoba. Salvo en los grupos de la provincia, familiarizados con el caso y el canal, en todos los otros, se consideraba que era poco clara, con poca producción, confusa y aburrida. De algún modo, esto parecía limitar el grado de empatía emocional con la víctima, si bien la temática estaba profundamente arraigada en todos los grupos. Como dijimos, con la noticia sobre corrupción del gobierno de Cristina F. de Kirchner en un canal opositor a su gobierno se señalaba una exageración en su presentación (demasiados movimientos de cámara, música alarmista). De este modo, ambas noticias, una por lo que se consideraba un bajo nivel de producción y la segunda por un exceso de intervención en su producción, parecían no respetar una gramática de las noticias de delito, afectando en un caso al entendimiento y a la empatía y en otro a la credibilidad.

Por lo demás, el noticiero es objeto de una crítica extendida en los grupos de clases bajas de la periferia de Buenos Aires. Lamentan la escasa presencia en las noticias de los problemas que azotan a sus barrios. En todos los grupos se vive como una marca de la desigualdad que los noticieros eligen presentar delitos menos graves de barrios más acomodados en lugar de aquellos que suceden en sus entornos y así, se preguntan si realmente le importa al resto de la sociedad la inseguridad en las zonas populares:

A veces a mí me llama la atención cómo hay veces que muestran noticias que por ahí no son tan graves, no sé, y vos decís acá a la vuelta mataron a un tipo de un negocio y no lo cuentan. Hay cosas que decís

Perspectivas de la Comunicación - Vol. 14 - No 1 - 2021 - pp. 139-168

Universidad de la Frontera - Chile 
¿cómo puede ser que no les haya llegado a los tipos esto que fue gravísimo? O sea, están cubriendo algo que no era tan relevante.

(M, NSE bajo, CABA 20-50)

Cabe destacar que esta diferencia no se veía en los grupos de las provincias, sin duda porque la menor cantidad de habitantes y hechos no hacía que rigiera un criterio de selección como en el área metropolitana. Las investigaciones locales han confirmado el hallazgo internacional de la mayor noticiabilidad de un crimen en la medida que se produce en las zonas y en víctimas más acomodadas, a pesar de la menor frecuencia de delitos (Palmieri \& Perelman, 2007). Las y los participantes debatían sobre las razones de lo que consideraban una "injusticia", como se revela en este intercambio en un grupo de Buenos Aires:

\author{
-Les parece que en general ¿el noticiero refleja la realidad en relación con el delito en sus \\ barrios o hablan de otras cosas? \\ H1: me parece que más que nada lo que sucede en Capital; Conurbano poco \\ M1: si no te enterás por internet, no te enterás \\ H2: son tendenciosos \\ H3: y... te da bronca \\ M2: Debería ser todo igual \\ H1: todos somos argentinos \\ (Grupo focal, NSE bajo, 30-50 años, GBA)
}

Esta crítica no los llevaba a descreer del noticiero, pero sí suscita reflexiones sobre lo que importa y lo que realmente genera agenda en los medios. Aparece como una dimensión de la desigualdad en la consideración pública que tiene consecuencias prácticas: si la situación de sus barrios no importa, tampoco habrá más despliegue de seguridad mediante la policía en esas zonas.

Ahora bien, también se miran noticias de delito por otras razones, algunas ya señaladas, otras como por cuestiones prácticas o pedagógicas, para poder saber qué pasó, qué zonas se han vuelto peligrosas y qué consejos darles a las hijas e hijos para estar atentos sobre nuevos delitos. Más en general, en los grupos de sectores populares, se miran noticias de inseguridad porque es un tema habitual y fácil de conversación. Al fin de cuentas, cada delito no precisa de datos de contexto o conocimientos adicionales, como sí sucede con noticias de política, economía o internacionales. 
Por ejemplo, yo le muestro esto que me acaban de pasar ustedes (noticia sobre corrupción), se lo muestro a alguien que no sabe nada y no entiende cuál es la noticia. Le muestro lo otro y sí entiende, bueno, hubo un chorro que vino, quiso matar, murió, le dispararon, murió. Eso es una noticia. Autoconclusión, empieza y termina, esa es la noticia. Acá no hay noticia, es un capítulo más y te hablo de lunes a viernes porque te perdés uno y cagaste.

(H, NSE bajo, GBA primer cordón 40-56)

La diferencia de clase era evidente: en los grupos de niveles medios hablar mucho de delitos tenía una connotación negativa, sin duda una marca de distinción de clase o nivel educativo, pero también de edad: son las y los "mayores" los que estarían "pegados al televisor" siguiendo la noticia de crímenes. Por último, algo novedoso observado en particular en algunos casos en grupos de sectores populares del Gran Buenos Aires y Rosario (los más preocupados por la inseguridad) es el interés por las noticias de delitos como un remedo de justicia y, en ciertos casos, de venganza y represalia. En efecto, la creencia subyacente para estos entrevistados es como la justicia actúa muy lentamente y se tarda en resolver, los noticieros al mostrar a los (presuntos) responsables, estarían haciendo las veces de un tribunal que tardará mucho o quizás nunca en conformarse y además estará al abrigo de la mirada pública.

Para mí el noticiero muestra su versión que él cree porque la justicia trabaja muy lentamente en la Argentina (H, NSE bajo, GBA 18-30)

Uno mira noticias para enterarse si se hace justicia o no...

(M, NSE bajo, GBA 40-56)

Así, para una parte importantes de los participantes que un noticiero exhiba a alguien como responsable de un hecho, muestre su rostro a la audiencia, lejos de ser considerado en forma negativa como un problema de presunción de culpabilidad y estigma, es juzgado positivamente. En efecto, ante la idea generalizada de que la mayoría de los crímenes terminan impunes, el noticiero hacía las veces de remedo de justicia, esto es, exhibía al sospechoso como posible culpable y, así, exponiéndolo, lo sometía al escarnio público como castigo.

¿Qué particularidades encontramos en los grupos de Rosario, Córdoba y Mendoza respecto del área metropolitana? En primer lugar, la estructura informativa y de recepción es un tanto diferente: como en las ciudades intermedias la gente suele volver a sus casas para el almuerzo, el noticiero del mediodía es importante y tiene mucha audiencia. En segundo 
lugar, los tiempos de viaje en transporte público son mucho más cortos que en el área metropolitana, por lo cual consumen menos noticias en su ir y venir cotidiano. En parte por esto, la idea del sinfín de noticias de delito estaba presente, pero con menos intensidad en estas ciudades, en particular en Mendoza, la menos populosa de estas tres. Sin embargo, comparado a un estudio en una ciudad intermedia (Cantú, 2002), donde los individuos tenían una rutina de consumo de noticias casi ritualizado, el cambio del ecosistema informativo ya ha llegado a todas las ciudades.

En todas las provincias hay dos noticieros locales de los dos canales más importantes. Estos exhiben algunas noticias de los noticieros nacionales y luego pasan a información local. En ciertos casos se mira un noticiero nacional y luego otro local, emitidos en forma consecutiva. Las y los participantes valoran sus noticieros, confían en ellos y las y los presentadores suelen ser personalidades de larga data y conocidos en sus ciudades, que participan también de otros programas de televisión o radio. Más aún, a veces expresan cierta familiaridad al referirse a los presentadores.

...hay gente que son más creíbles que otras. Por falta de edad o por falta de credibilidad. Yo, por ejemplo,
como para hacer nombres, al Turco yo le creo, a pesar de que yo estoy con la radio, estoy con el
WhatsApp y por ahí se lo mando y le digo para el Turco que le escribís porque tenés la posibilidad y él
te lo lee, y entonces vos decís bueno, te enteraste que yo tengo una manera diferente de pensar que vos
pero que yo piense diferente que vos no me hace tu enemigo. (H, NSE bajo, Mendoza, 25-55)

Los noticieros son diferentes por la gente que los conduce. Los conocemos desde hace años...

(M, NSE bajo, Córdoba, 25-55)

De este modo, al haber menos tiempo de consumo disponible, menos información de delitos, la cantidad de información acumulada a lo largo del día parecía menor, sobre todo en Mendoza. No obstante, el rol organizador se mantenía, pero también aparecían otras dos funciones que no observamos en los noticieros del área metropolitana. Una, es que los noticieros locales refuerzan la identidad provincial: presentan noticias de los distintos departamentos o municipios, festividades o actividades culturales o deportivas, difunden información positiva sobre la provincia como por ejemplo logros en deportes, artes, ciencias. La segunda, los informativos locales son canales de comunicación y expresión de demandas y reclamos, lo cual fue particularmente valorado en los grupos de sectores populares de las 3 ciudades y fue un tema casi ausente en los grupos de sectores medios. En efecto, los 
noticieros tienen números de teléfono y WhatsApp para que la gente se comunique. Así, corte de servicios, calles rotas, demoras en ser atendido en un hospital y otras vicisitudes cotidianas son enviadas a los medios para intentar que los responsables políticos o las empresas privadas se vean interpeladas y las resuelvan. No siempre tienen éxito, pero al menos aparece como el único canal para ser escuchado.

Para finalizar, retomamos un tema clásico: el impacto de las noticias de delitos del área metropolitana en las ciudades de menor talla. El trabajo sobre noticias locales en periódicos nacionales de Estados Unidos, "sintiéndose seguro por comparación" demostraba que la lectura de noticias de crímenes de otras ciudades afectaba a grupos sociales o étnicos distintos al propio, lo que generaba o reforzaba una sensación de seguridad local frente a otras urbes (Liska \& Baccaglini, 1990). En nuestro trabajo en Argentina hace una década (Kessler, 2009) mostramos algo diferente respecto de las noticias televisivas. Los delitos difundidos por canales de noticias con sede en Buenos Aires lejos de contribuir a una sensación de seguridad local, agudizaba una incipiente preocupación por el crimen. Se temía una supuesta "llegada" de delincuentes desde el área metropolitana hacia otros lugares, "olas de delito" que se expandirían y, en las urbes más alejadas, como una suerte de anticipación de un proceso de degradación social que, tarde o temprano, también pasará. Los grupos fueron realizados 8 años más tarde y mostraban una situación distinta en cada ciudad. Rosario tiene una tasa de delito muy alta y una gran preocupación por el crimen ligado al narcotráfico local, que de hecho se ha transformado en un problema público nacional. Aunque en los dos grupos se manifestaba una gran preocupación, por otro lado, les molestaba la presencia de esas noticias en los informativos nacionales porque incidía en dar una muy mala imagen de la ciudad al resto del país. Por su parte, en Córdoba y Mendoza juzgaban que la situación de inseguridad en Buenos Aires era peor, sin embargo, no se sentían "seguros por comparación", a lo sumo consideraban una gradación menor en la gravedad del problema que en el área metropolitana. De todos modos, en ambas ciudades, había entrevistados que estimaban una exageración de la inseguridad en los noticieros respecto de la realidad local.

Tengo una idea de que en todos lados suceden cosas, pero el nivel de psicosis que generan acá en Mendoza es aterrador, porque si en Estados Unidos tienen un nivel de vida alto, sin embargo, tienen una tasa de delincuencia mucho más alta por la cantidad de habitantes, una inversión enorme en dinero. Y acá cada vez que prendés el noticiero alguien se murió, nos robaron...

(H, NSE medio, Mendoza 25-55)

Perspectivas de la Comunicación - Vol. 14 - No 1 - 2021 - pp. 139-168

Universidad de la Frontera - Chile 
La diferencia central es que, comparado al trabajo realizado en 2009, la idea del contagio o la extensión no estaba, porque efectivamente ya había sucedido: los estudios muestran que en todas las ciudades intermedias y aún pequeñas el tema de la inseguridad es un problema público estabilizado, esto es, una rúbrica mediática, objeto de políticas y de controversias habituales.

\section{Discusión}

En este artículo trabajamos acerca de las nuevas formas de consumo de noticias y el modo en que el noticiero se configura como ordenador de la información. Consideramos que trabajar con grupos focales como metodología tiene fortalezas y debilidades. Entre las primeras consideramos que los grupos permitieron observar de manera más clara que en las entrevistas individuales las interacciones entre los participantes en relación con el consumo de medios. Por supuesto, se trata de un universo muestral acotado, por lo cual, las conclusiones de este trabajo podrían dar lugar a hipótesis a ser retomadas en futuras investigaciones. A su vez, se trata de una realidad tan cambiante que periódicamente deberíamos revalidar los hallazgos.

Así, en el artículo propusimos un recorrido con el fin de analizar las nuevas formas de interacción mediática y el lugar del noticiero en este entramado. En primer lugar, mostramos los modos en que las personas se informan en la actualidad, predominantemente entre portales digitales, redes y televisión. El uso de dispositivos móviles es el cambio más trascendental a la hora de analizar las prácticas, tal como mostramos con el uso del tiempo de viaje para esta actividad. También corroboramos la extensión del consumo incidental, que ya muestra la literatura sobre el tema, pero avanzamos en relación con posibles caminos que pueden tomarse una vez que las noticias "se les aparecen" a los usuarios. En este punto, las noticias de delito y de inseguridad mostraron ser mediadoras en lo que denominamos del consumo incidental al activo. Ahora bien, una vez que los usuarios tienen intenciones de informarse, buscan información desde distintas fuentes (incluso que siguen líneas editoriales opuestas) con el fin de configurar sus propias noticias. Estas prácticas que desarrollan los sujetos están vinculadas con el crecimiento de las fake news, y de la desinformación en general, sumado en el caso local, a un escenario mediático polarizado. Luego de este primer recorrido por las nuevas formas de consumo, nos preguntamos por el lugar del noticiero como institución, y allí encontramos que las personas mantienen un contrato de lectura con este género. Algunas cuestiones que se desprenden de las entrevistas permiten mostrar que, en la vorágine informativa, el noticiero funciona como ordenador. 
Las noticias en la pantalla y con la marca del noticiero renuevan un pacto de credibilidad que acompaña la crisis general de confianza en los medios, que como muestran distintos estudios, es muy alta en Argentina. Aun así, el noticiero ha cambiado en la forma de presentar las noticias, incluso con la incorporación de nuevas secciones y de distintos presentadores, lo que no le ha quitado veracidad. En general los noticieros de aire se mantienen alejados de una mirada partisana de las noticias, y su credibilidad está en parte determinada, como mostramos, por los modos de presentación de las noticias. Para M. Grillo (2007) los noticieros deben ser creíbles y, al mismo tiempo, mantener su público. Por esto, "deben seguir los cánones de construcción de la verosimilitud sin perder de vista que necesitan lograr y mantener una audiencia” (p. 131). Esta necesidad de ordenar con credibilidad se hace más intensa en el caso de las noticias de delito, dada la alta cantidad de información que circula sobre el tema y la gran preocupación que promueve. En efecto, ya sea por posteo, grupos de WhatsApp, noticias sensacionalistas o con "morbo" la vorágine de noticias sobre delitos genera sin duda preocupación, quizás angustias y, por eso, la necesidad de ordenar, priorizar y descartar que permiten hacer diariamente los noticieros de la noche es particularmente importante. Una tarea y un “sin fin” que recomenzará al día siguiente.

\section{Conflicto de interés}

Los autores declaran que no existe conflicto de interés. 


\section{Referencias bibliográficas}

ALGHADIR, A., GABR, S. \& IQBAL, Z. (2016): Television watching, diet and body mass index of school children in Saudi Arabia. Pediatrics Internacional, 58(4), pp. 290-294.

AMADO, A. \& RINCÓN, O. (2015): La reinvención de los discursos o cómo entender a los bárbaros del siglo XXI en la comunicación en mutación. En A. Amado y O. Rincón (Eds.), La comunicación en mutación (pp. 5-12). Bogotá, Fundación Friedrich Ebert (FES).

ANDREWS, K. T. \& CAREN, N. (2010): Making the news: Movement organizations, media attention, and the public agenda. American Sociological Review 75(6), pp. 841-866.

BAYARMA, A., ETTEMA, D. \& DIJST, M. (2010): Fragmentation of work activity as a multidimensional construct and its association with ICT, employment and sociodemographic characteristics. Journal of Transport Geography, 18(1), pp. 55-64.

BECERRA, M. \& MARINO, S. (2021): La tv abierta de las grandes ciudades argentinas: estructura subordinada y contenido local. En N. Anselmino, N. Aruguete, M. Becerra y G. Kessler (Eds.), El delito televisado. Buenos Aires, Biblos.

BERGSTRÖM, A. \& JERVELYCKE, M. (2018): News in Social Media. Incidential Consumption and the Role of Opinion Leaders. Digital Journalism, 6(5), pp. 583-598.

BERRY, J. M., \& SOBIERAJ, S. (2014): The outrage industry: Political opinion media and the new incivility. Oxford (England), Oxford University Press.

BOCZKOWSKI, P. \& MITCHELSTEIN, E. (2013): The News Gap. When the information Preferences of the Media and Public Diverge. Massachusetts, The Mit Press.

BOCZKOWSKI, P., MITCHELSTEIN, E., \& MATASSI, M. (2017). Incidental news: How young people consume news on social media. Proceedings of the 5oth Hawaii international conference on system sciences.

https://scholarspace.manoa.hawaii.edu/handle/10125/41371 
CANTÚ, A. (2002): Detrás de las noticias. Una mirada desde los consumidores de información. Oficios Terrestres, 11-12, pp. 111-121.

(5, 6 y 7 de junio de 2013): ¿Qué ves cuando me ves? El noticiero televisivo desde la mirada de los televidentes [Sesión de Conceferencia]. VI Encuentro Panamericano de Comunicación (ECI-UNC), Córdoba, Argentina.

FEEZELL, J. T. (2018): Agenda setting through social media: The importance of incidental news exposure and social filtering in the digital era. Political Research Quarterly, 71(2), pp. 482-494.

FERRANTE, P. (29 y 30 de agosto de 2019): TV en movimiento: Experiencias de consumo de contenidos audiovisuales en el subte de Bs.As. [Sesión de Congreso]. Quinto Congreso Anual Desarrollos Contemporáneos Sobre Medios, Cultura y Sociedad: Argentina y América Latina, Buenos Aires, Argentina.

FOCAS, B. (2020): El delito y sus públicos. Inseguridad, medios y polarización. Buenos Aires, Unsam-edita.

GAYOL, S. \& KESSLER, G. (2018): Muertes que importan. Una mirada sociohistórica sobre los casos que marcaron la argentina reciente. Buenos Aires, Siglo XXI.

GOU, L. (2014): Toward the Third Level of Agenda Setting Theory. En T. J. Johnson (Ed.), Agenda Setting in a 2.o World (pp. 112-133). Nueva York, Routledge.

GRABE, M. E., ZHOU, S. \& BARNETT, B. (2001): Explicating Sensationalism in Television News: Content and the Bells and Whistles of Form. Journal of Broadcasting \& Electronic Media, 45(4), pp. 635-655.

GRILlO, M. (2007): El público en los noticieros televisivos. Conexão - Comunicação e Cultura, 6(11), pp. 123-138.

GUO, Z., DERIAN, A. \& ZHAO, J. (2015): Smart Devices and Travel Time Use by Bus Passengers in Vancouver, Canada. International Journal of Sustainable Transportation, 9(5), pp. 335-347. 
HARDER, R. A., SEVENANS, J. \& VAN-AELST, P. (2017): Intermedia agenda setting in the social media age: How traditional players dominate the news agenda in election times. The International Journal of Press/Politics, 22(3), pp.275-293.

JULSRUD, T. \& DENSTADLI, J. (2017): Smartphones, travel time-use and attitudes to public transport services Insights from an explorative study of urban dwellers in two Norwegian cities. International Journal of Sustainable Transportation, 11(8), pp. 602-610.

KENYON, S. \& LYONS, G. (2007): Introducing multitasking to the study of travel and ICT: examining its extent and assesing its potential importance. Transportation Research Part A: Policy and Practice, 41(2), pp. 161-175.

KESSLER, G. \& FOCÁS, B. (2014): ¿Responsables del temor? Medios y sentimiento de inseguridad en América Latina. Nueva Sociedad, 249, pp. 137-148.

KESSLER, G. (2009): El sentimiento de inseguridad. Sociología del temor al delito. Buenos Aires, Siglo XXI.

LEE, E. \& TANDOC, E. (2017): When News Meets the Audience: How Audience Feedback Online Affects News Production and Consumption. Human communication research, 43(4), pp. 436-449. https://doi.org/10.1111/hcre.12123

LENZ, B. \& NOBIS, C. (2007): The changing allocation of activities in space and time by the use of ICT - "Fragmentation" as a new concept and empirical results. Transportation Research Part A: Policy and Practice, 41(2), pp. 190-204.

LEVY, M. R. (1977): Experiencing television news. Journal of Communication 27(4), pp. 112-117.

LISKA, A. E. \& BACCAGLINI, W. (1990): Feeling safe by comparison: Crime in the newspapers. Social Problems, 37(3), pp. 360-374. 
LYONS, G. \& URRY, J. (2005): Travel time use in the information age. Transportation Research Part A: Policy and Practice, 39(2-3), pp. 257-276.

MCCOMBS, M. \& BARRIOS, M. (2012): Evolución de los Tres Niveles de la Teoría de Agenda-Setting: El impacto de las noticias en el público. En M. McCombs y M. Martín (Eds.), Communication and Social Life: Studies in honor of profesor Esteban López-Escobar (pp. 579 - 591). Pamplona (España), EUNSA.

MCCOMBS, M. (2007): Estableciendo la agenda. El impacto de los medios en la opinión pública y en el conocimiento. Barcelona, Paidós.

MITCHELSTEIN, E. \& BOCZKOWSKI, P. (2017): Juventud, estatus y conexiones. Explicación del consumo incidental de noticias en redes sociales. Revista Mexicana de Opinión Pública, 13(24), pp. 131-145.

MORLEY, D. (1996): Televisión, audiencias y estudios culturales. Madrid, Amorrortu Editores.

NELSON, J. \& LEI, R. F. (2018): The Effect of Digital Platforms on News Audience Behavior. Digital Journalism, 6(5), pp. 619-633.

PAJONI, H. (2012): Noticieros en Argentina: cerca del espectáculo, lejos de la política. Cuadernos de información, (31), pp. 129-138.

PALMIERI, G. \& PERELMAN, M. (2007): Olas de inseguridad. Actores, discursos y políticas en un debate público que atraviesa derechos humanos y exclusión. En Centro de Estudios Legales y Sociales (CELS), Derechos Humanos en Argentina. Informe 2007 (pp. 223-241). Buenos Aires, Siglo XXI.

PONCE, C. L. (2015): That's the Way it is. A History of Television News in America. Illinois (EE.UU.), The University of Chicago Press. 
RAEYMAECKERS, K. (2002): Research Note: Young People and Patterns of Time Consumption in Relation to Print Media. European Journal of Communication, 17(3), pp. 369-383.

SILVERSTONE, R. (1996): Televisión y vida cotidiana. Buenos Aires, Amorrortu ediciones. (2004): ¿Por qué estudiar los medios? Buenos Aires, Amorrortu.

THURMAN, N. (2017): Newspaper Consumption in the Mobile Age: Re-assessing multiplatform performance and market share using 'time-spent'. Journalism Studies, 19(10), pp. 1409-1429. DOI: 10.1080/1461670X.2017.1279028

URRY, J. (2007): Mobilities. Cambridge, Polity.

WEBSTER, J. G. (2005): Beneath the veneer of fragmentation: Television audience polarization in a multichannel world. Journal of Communication, 55, pp. 366-382.

(2014): The marketplace of attention: How audiences take shape in a digital age. Massachusetts, The Mit Press

WIELAND, M. \& KLEINEN-VON-KÖNIGSLÖW, K. (2020): Conceptualizing different forms of news processing following incidental news contact: A triple path model. Journalism, 21(8), pp. 1049-1066. https://doi.org/10.1177/1464884920915353

YORKE, I. (2017): Television news. Londres, Routledge. 